\title{
Some new Pascal sequence spaces
}

\author{
Harun Polat ${ }^{a^{*}}$ \\ ${ }^{a}$ Department of Mathematics, Faculty of Science and Arts, Muş Alparslan University, Muş, Turkey \\ *Corresponding author E-mail: h.polat@alparslan.edu.tr
}

\section{Article Info}

Keywords: $\alpha$-, $\beta$ - and $\gamma$-duals and basis of sequence, Matrix mappings, Pascal sequence spaces

2010 AMS: 46A35, 46A45, 46B45

Received: 27 March 2018

Accepted: 25 June 2018

Available online: 30 June 2018

\begin{abstract}
The main purpose of the present paper is to study of some new Pascal sequence spaces $p_{\infty}, p_{c}$ and $p_{0}$. New Pascal sequence spaces $p_{\infty}, p_{c}$ and $p_{0}$ are found as $B K$-spaces and it is proved that the spaces $p_{\infty}, p_{c}$ and $p_{0}$ are linearly isomorphic to the spaces $l_{\infty}, c$ and $c_{0}$ respectively. Afterward, $\alpha-, \beta$ - and $\gamma$-duals of these spaces $p_{c}$ and $p_{0}$ are computed and their bases are consructed. Finally, matrix the classes $\left(p_{c}: l_{p}\right)$ and $\left(p_{c}: c\right)$ have been characterized.
\end{abstract}

\section{Preliminaries, background and notation}

By $w$, we shall denote the space all real or complex valued sequences. Any vector subspace of $w$ is called a sequence space. We shall write $l_{\infty}, c$, and $c_{0}$ for the spaces of all bounded, convergent and null sequence are given by $l_{\infty}=\left\{x=\left(x_{k}\right) \in w: \sup _{k \rightarrow \infty}\left|x_{k}\right|<\infty\right\}$, $c=\left\{x=\left(x_{k}\right) \in w: \lim _{k \rightarrow \infty} x_{k}\right.$ exists $\}$ and $c_{0}=\left\{x=\left(x_{k}\right) \in w: \lim _{k \rightarrow \infty} x_{k}=0\right\}$. Also by $b s, c s, l_{1}$ and $l_{p}$ we denote the spaces of all bounded, convergent, absolutely convergent and $p$-absolutely convergent series, respectively.

A sequence space $\lambda$ with a linear topology is called an $K$-space provided each of the maps $p_{i}: \lambda \rightarrow \mathbb{C}$ defined by $p_{i}(x)=x_{i}$ is continuous for all $i \in \mathbb{N}$; where $\mathbb{C}$ denotes the set of complex field and $\mathbb{N}=\{0,1,2, \ldots\}$. An $K$-space $\lambda$ is called an $F K$-space provided $\lambda$ is a complete linear metric space. An $F K$-space provided whose topology is normable is called a $B K$ - space [1].

Let $X, Y$ be any two sequence spaces and $A=\left(a_{n k}\right)$ be an infinite matrix of real numbers $a_{n k}$, where $n, k \in \mathbb{N}$. Then, we write $A x=\left((A x)_{n}\right)$, the $A$-transform of $x$, if $A_{n}(x)=\sum_{k} a_{n k} x_{k}$ converges for each $n \in \mathbb{N}$. If $x \in X$ implies that $A x \in Y$, then we say that $A$ defines a matrix transformation from $X$ into $Y$ and denote it by $A: X \rightarrow Y$. By $(X: Y)$ we denote the class of all infinite matrices $A$ such that $A: X \rightarrow Y$. For simplicity in notation, here and in what follows, the summation without limits runs from 0 to $\infty$.

Let $F$ denote the collection of all finite subsets on $\mathbb{N}$ and $K, \mathbb{N} \subset F$. The matrix domain $X_{A}$ of an infinite matrix $A$ in a sequence space $X$ is defined by

$$
X_{A}=\left\{x=\left(x_{k}\right) \in w: A x \in X\right\}
$$

which is a sequence space.

The approach constructing a new sequence space by means of the matrix domain of a particular limitation method was used by authors $[2,3,4,5,6,7,8]$. They introduced the sequence spaces $\left(c_{0}\right)_{T^{r}}=t_{0}^{r}$ and $(c)_{T^{r}}=t_{c}^{r}$ in [2], $\left(c_{0}\right)_{E^{r}}=e_{0}^{r}$ and $(c)_{E^{r}}=e_{c}^{r}$ in [3], $\left(c_{0}\right)_{C}=\bar{c}_{0}$ and $c_{C}=\bar{c}$ in [4], $\left(l_{p}\right)_{E^{r}}=e_{p}^{r}$ in [5], $\left(l_{\infty}\right)_{R^{t}}=r_{\infty}^{t}, c_{R^{t}}=r_{c}^{t}$ and $\left(c_{0}\right)_{R^{t}}=r_{0}^{r}$ in [6], $\left(l_{p}\right)_{C}=X_{p}$ in [7] and $\left(l_{p}\right)_{N_{q}}$ in [8] where $T^{r}, E^{r}, C, R^{t}$ and $N_{q}$ denote the Taylor, Euler, Cesaro, Riesz and Nörlund means, respectively.

Following $[2,3,4,5,6,7,8]$, this way, the purpose of this paper is to introduce the new Pascal sequence spaces $p_{\infty}, p_{c}$ and $p_{0}$ and derive some results related to those sequence spaces. Furthermore, we have constructed the basis and computed the $\alpha$-, $\beta$ - and $\gamma$-duals of the spaces $p_{\infty}, p_{c}$ and $p_{0}$. Finally, we have characterized the matrix mappings from the space $p_{c}$ to $l_{p}$ and from the space $p_{c}$ to $c$. 


\section{The Pascal matrix of inverse formula and Pascal sequence spaces}

Let $P$ denote the Pascal means defined by the Pascal matrix [9] as is defined by

$$
P=\left[p_{n k}\right]=\left\{\begin{array}{c}
\left(\begin{array}{c}
n \\
n-k
\end{array}\right),(0 \leq k \leq n) \\
0,(k>n)
\end{array},(n, k \in \mathbb{N})\right.
$$

and the inverse of Pascal's matrix $P_{n}=\left[p_{n k}\right][10]$ is given by

$$
P^{-1}=\left[p_{n k}\right]^{-1}=\left\{\begin{array}{c}
(-1)^{n-k}\left(\begin{array}{c}
n \\
n-k
\end{array}\right),(0 \leq k \leq n) \\
0,(k>n)
\end{array},(n, k \in \mathbb{N}) .\right.
$$

There is some interesting properties of Pascal matrix. For example; we can form three types of matrices: symmetric, lower triangular, and upper triangular, for any integer $n>0$. The symmetric Pascal matrix of order $n$ is defined by

$$
S_{n}=\left(s_{i} j\right)=\left(\begin{array}{c}
i+j-2 \\
j-1
\end{array}\right) i, j=1,2, \ldots, n .
$$

We can define the lower triangular Pascal matrix of order $n$ by

$$
L_{n}=\left(l_{i j}\right)=\left\{\begin{array}{cc}
\left(\begin{array}{c}
i-1 \\
j-1
\end{array}\right), & (0 \leq j \leq i) \\
0, & (j>i)
\end{array},\right.
$$

and the upper triangular Pascal matrix of order $n$ is defined by

$$
U_{n}=\left(u_{i j}\right)=\left\{\begin{array}{cc}
\left(\begin{array}{c}
j-1 \\
i-1
\end{array}\right), & (0 \leq i \leq j) \\
0, & (j>i)
\end{array}\right.
$$

We notice that $U_{n}=\left(L_{n}\right)^{T}$, for any positive integer $n$.

i. Let $S_{n}$ be the symmetric Pascal matrix of order $n$ defined by (2.1), $L_{n}$ be the lower triangular Pascal matrix of order $n$ defined by (2.3), and $U_{n}$ be the upper triangular Pascal matrix of order $n$ defined by (2.4), then $S_{n}=L_{n} U_{n}$ and $\operatorname{det}\left(S_{n}\right)=1$ [11].

ii. Let $A$ and $B$ be $n \times n$ matrices. We say that $A$ is similar to $B$ if there is an invertible $n \times n$ matrix $P$ such that $P^{-1} A P=B$ [12].

iii. Let $S_{n}$ be the symmetric Pascal matrix of order $n$ defined by (2.2), then $S_{n}$ is similar to its inverse $S_{n}^{-1}$ [11].

iv. Let $L_{n}$ be the lower triangular Pascal matrix of order $n$ defined by $(2.3)$, then $L_{n}^{-1}=\left((-1)^{i-j} l_{i j}\right)[13]$.

We wish to introduce the Pascal sequence spaces $p_{\infty}, p_{c}$ and $p_{0}$, as the set of all sequences such that $P$-transforms of them are in the spaces $l_{\infty}, c$ and $c_{0}$, respectively, that is

$$
\begin{aligned}
& p_{\infty}=\left\{x=\left(x_{k}\right) \in w: \sup _{n}\left|\sum_{k=0}^{n}\left(\begin{array}{c}
n \\
n-k
\end{array}\right) x_{k}\right|<\infty\right\}, \\
& p_{c}=\left\{x=\left(x_{k}\right) \in w: \lim _{n \rightarrow \infty} \sum_{k=0}^{n}\left(\begin{array}{c}
n \\
n-k
\end{array}\right) x_{k} \text { exists }\right\}
\end{aligned}
$$

and

$$
p_{0}=\left\{x=\left(x_{k}\right) \in w: \lim _{n \rightarrow \infty} \sum_{k=0}^{n}\left(\begin{array}{c}
n \\
n-k
\end{array}\right) x_{k}=0\right\} .
$$

With the notation of (1.1), we may redefine the spaces $p_{\infty}, p_{c}$ and $p_{0}$ as follows:

$$
p_{\infty}=\left(l_{\infty}\right)_{P}, p_{c}=(c)_{P} \text { and } p_{0}=\left(c_{0}\right)_{P}
$$

If $\lambda$ is an normed or paranormed sequence space, then matrix domain $\lambda_{P}$ is called an Pascal sequence space. We define the sequence $y=\left(y_{n}\right)$ which will be frequently used, as the $P$-transform of a sequence $x=\left(x_{n}\right)$ i.e.,

$$
y_{n}=\sum_{k=0}^{n}\left(\begin{array}{c}
n \\
n-k
\end{array}\right) x_{k}, \quad(n \in N) .
$$

It can be shown easily that $p_{\infty}, p_{c}$ and $p_{0}$ are linear and normed spaces by the following norm:

$$
\|x\|_{p_{0}}=\|x\|_{p_{c}}=\|x\|_{p_{\infty}}=\|P x\|_{l_{\infty}} .
$$

Theorem 2.1. The sequence spaces $p_{\infty}, p_{c}$ and $p_{0}$ endowed with the norm (2.7) are Banach spaces. 
Proof. Let sequence $\left\{x^{t}\right\}=\left\{x_{0}^{(t)}, x_{1}^{(t)}, x_{2}^{(t)}, \ldots\right\}$ at $p_{\infty}$ a Cauchy sequence for every fixed $t \in \mathbb{N}$. Then, there exists an $n_{0}=n_{0}(\varepsilon)$ for every $\varepsilon>0$ such that $\left\|x^{t}-x^{r}\right\|_{\infty}<\varepsilon$ for all $t, r>n_{0}$. Hence, $\left|P\left(x^{t}-x^{r}\right)\right|<\varepsilon$ for all $t, r>n_{0}$ and for each $k \in \mathbb{N}$.

Therefore, $\left\{P x_{k}^{t}\right\}=\left\{\left(P x^{0}\right)_{k},\left(P x^{1}\right)_{k},\left(P x^{2}\right)_{k}, \ldots\right\}$ is a Cauchy sequence in the set of complex numbers $\mathbb{C}$. Since $\mathbb{C}$ is complete, it is convergent say $\lim _{t \rightarrow \infty}\left(P x^{t}\right)_{k}=(P x)_{k}$ and $\lim _{m \rightarrow \infty}\left(P x^{m}\right)_{k}=(P x)_{k}$ for each $k \in \mathbb{N}$. Hence, we have

$$
\lim _{m \rightarrow \infty}\left|P x_{k}^{t}-x_{k}^{m}\right|=\left|P\left(x_{k}^{t}-x_{k}\right)-P\left(x_{k}^{m}-x_{k}\right)\right| \leq \varepsilon \text { for all } n \geq n_{0} .
$$

This implies that $\left\|x^{t}-x^{m}\right\| \rightarrow \infty$ for $t, m \rightarrow \infty$. Now, we should that $x \in p_{\infty}$. We have

$$
\begin{gathered}
\|x\|_{\infty}=\|P x\|_{\infty}=\sup _{n}\left|\sum_{k=0}^{n}\left(\begin{array}{c}
n \\
n-k
\end{array}\right) x_{k}\right|=\sup _{n} \mid \sum_{k=0}^{n}\left(\begin{array}{c}
n \\
n-k
\end{array}\right)\left(x_{k}-x_{k}^{t}+x_{k}^{t} \mid\right. \\
\leq \sup _{n}\left|P\left(x_{k}^{t}-x_{k}\right)\right|+\sup _{n}\left|P x_{k}^{t}\right| \\
\leq\left\|x^{t}-x\right\|_{\infty}+\left|P x_{k}^{t}\right|<\infty
\end{gathered}
$$

for $t, k \in \mathbb{N}$. This implies that $x=\left(x_{k}\right) \in p_{\infty}$. Thus, $p_{\infty}$ the space is a Banach space with the norm (2.7). It can be shown that $p_{0}$ and $p_{c}$ are closed subspaces of $p_{\infty}$ which leads us to the consequence that the spaces $p_{0}$ and $p_{c}$ are also the Banach spaces with the norm (2.7). Furthermore, since $p_{\infty}$ is a Banach space with continuous coordinates, i.e., $\left\|P\left(x_{k}^{t}-x\right)\right\|_{\infty} \rightarrow \infty$ imples $\left|P\left(x_{k}^{t}-x_{k}\right)\right| \rightarrow \infty$ for all $k \in \mathbb{N}$, it is also a $B K$-space.

Theorem 2.2. The sequence spaces $p_{\infty}, p_{c}$ and $p_{0}$ are linearly isomorphic to the spaces $l_{\infty}, c$ and $c_{0}$ respectively, i.e $p_{\infty} \cong l_{\infty}, p_{c} \cong c$ and $p_{0} \cong c_{0}$.

Proof. To prove the fact $p_{0} \cong c_{0}$, we should show the existence of a linear bijection between the spaces $p_{0}$ and $c_{0}$. Consider the transformation $T$ defined, with the notation (2.6), from $p_{0}$ to $c_{0}$. The linearity of $T$ is clear. Further, it is trivial that $x=0$ whenever $T x=0$ and hence $T$ is injective.

Let $y \in c_{0}$. We define the sequence $x=\left(x_{k}\right)$ as follows:

$$
x_{k}=\sum_{i=0}^{k}(-1)^{k-i}\left(\begin{array}{c}
k \\
k-i
\end{array}\right) y_{i}
$$

Then

$$
\lim _{n \rightarrow \infty}(P x)_{n}=\lim _{n \rightarrow \infty} \sum_{k=0}^{n}\left(\begin{array}{c}
n \\
n-k
\end{array}\right) \sum_{i=0}^{k}(-1)^{k-i}\left(\begin{array}{c}
k \\
k-i
\end{array}\right) y_{i}=\lim _{n \rightarrow \infty} y_{n}=0 .
$$

Thus, we have that $x \in p_{0}$. In addition, note that

$$
\|x\|_{p_{0}}=\sup _{n \in \mathbb{N}}\left|\sum_{k=0}^{n}\left(\begin{array}{c}
n \\
n-k
\end{array}\right) \sum_{i=0}^{k}(-1)^{k-i}\left(\begin{array}{c}
k \\
k-i
\end{array}\right) y_{i}\right|=\sup _{n \in \mathbb{N}}\left|y_{n}\right|=\|y\|_{c_{0}}<\infty .
$$

Consequently, $T$ is surjective and is norm preserving. Hence, $T$ is a linear bijection which therefore says us that the spaces $p_{0}$ to $c_{0}$ are linearly isomorphic. In the same way, it can be shown that $p_{c}$ and $p_{\infty}$ are linearly isomorphic to $c$ and $l_{\infty}$, respectively, and so we omit the detail.

Before giving the basis of of the sequence spaces $p_{c}$ and $p_{0}$, we define the Schauder basis. A sequence $\left(b_{n}\right)_{n \in \mathbb{N}}$ in a normed sequence space $\lambda$ is called a Schauder basis (or briefly basis) [14], if for every $x \in \lambda$ there is a unique sequence $\left(\alpha_{n}\right)$ of scalars such that

$$
\lim _{n \rightarrow \infty}\left\|x-\left(\alpha_{0} x_{0}+\alpha_{1} x_{1}+\ldots+\alpha_{n} x_{n}\right)\right\|=0
$$

In the following theorem, we shall give the Schauder basis for the spaces $p_{c}$ and $p_{0}$.

Theorem 2.3. Let $k \in \mathbb{N}$ a fixed natural number and $b^{(k)}=\left\{b_{n}^{(k)}\right\}_{n \in \mathbb{N}}$ where

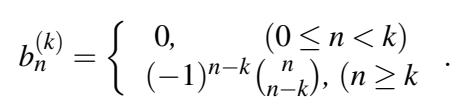

Then the following assertions are true:

$i$. The sequence $\left\{b_{n}^{(k)}\right\}$ is a basis for the space $p_{0}$ and every $x \in p_{0}$ has a unique representation of the from $x=\sum_{k} \lambda_{k} b^{(k)}$ where $\lambda_{k}=(P x)_{k}$ for all $k \in \mathbb{N}$.

ii. The set $\left\{e, b^{(0)}, b^{(1)}, \ldots, b^{(k)}, \ldots\right\}$ is a basis for the space $p_{c}$ and every $x \in p_{c}$ has a unique representation of the form $x=$ le + $\sum_{k}\left(\lambda_{k}-l\right) b^{(k)}$, where $l=\lim _{k \rightarrow \infty}(P x)_{k}$ and $\lambda_{k}=(P x)_{k}$ for all $k \in \mathbb{N}$. 


\section{The $\alpha-, \beta-$ and $\gamma-$ duals of the spaces $p_{\infty}, p_{c}$ and $p_{0}$}

In this section, we state and prove the theorems determining the $\alpha$-, $\beta$ - and $\gamma$-duals of the sequence spaces $p_{\infty}, p_{c}$ and $p_{0}$. For the sequence spaces $X$ and $Y$ define the set $S(X, Y)$ by

$$
S(X, Y)=\left\{z=\left(z_{k}\right) \in w: x z=\left(x_{k} z_{k}\right) \in Y \text { for all } x \in X\right\} .
$$

The $\alpha-, \beta$ - and $\gamma$-duals of the sequence spaces $\lambda$, which are respectively denoted by $\lambda^{\alpha}, \lambda^{\beta}$ and $\lambda^{\gamma}$ are defined by Garling [15] , by $\lambda^{\alpha}=S\left(\lambda, l_{1}\right), \lambda^{\beta}=S(\lambda, c s)$ and $\lambda^{\gamma}=S(\lambda, b s)$. We shall begin with the Lemmas due to Stieglitz and Tietz [16], which are needed in the proof of the Theorems 3.4-3.6.

Lemma 3.1. $A \in\left(c_{0}: l_{1}\right)=\left(c: l_{1}\right)$ if and only if

$$
\sup _{K \in F} \sum_{n}\left|\sum_{k \in K} a_{n k}\right|<\infty
$$

Lemma 3.2. $A \in\left(c_{0}: c\right)$ if and only if

$$
\begin{gathered}
\sup _{n} \sum_{k}\left|a_{n k}\right|<\infty, \\
\lim _{n \rightarrow \infty} a_{n k}=\alpha_{k},(k \in \mathbb{N}) .
\end{gathered}
$$

Lemma 3.3. $A \in\left(c_{0}: l_{\infty}\right)$ if and only if (3.2) holds.

Theorem 3.4. The $\alpha$-dual of the sequence spaces $p_{\infty}, p_{c}$ and $p_{0}$ is the set

$$
D=\left\{a=\left(a_{k}\right) \in w: \sup _{K \in F} \sum_{n}\left|\sum_{k \in K}(-1)^{n-k}\left(\begin{array}{c}
n \\
n-k
\end{array}\right) a_{n}\right|<\infty\right\} .
$$

Proof. Let $a=\left(a_{n}\right) \in w$ and consider the matrix $B$ whose rows are the products of the rows of the matrix $P^{-1}$ and sequence $a=\left(a_{n}\right)$. Bearing in mind the relation (2.3), we immediately derive that

$$
a_{n} x_{n}=\sum_{k=0}^{n}(-1)^{n-k}\left(\begin{array}{c}
n \\
n-k
\end{array}\right) a_{n} y_{k}=\sum_{k=0}^{n} b_{n k} y_{k}=(B y)_{n},(n \in \mathbb{N}) .
$$

Therefore by (3.4) we observe that that $a x=\left(a_{n} x_{n}\right) \in l_{1}$ whenever $x \in p_{\infty}, p_{c}$ and $p_{0}$ if and only if $B y \in l_{1}$ whenever $y \in l_{\infty}, c$, and $c_{0}$. Then, we derive by Lemma 3.1 that

$$
\sup _{K \in F} \sum_{n}\left|\sum_{k \in K}(-1)^{n-k}\left(\begin{array}{c}
n \\
n-k
\end{array}\right) a_{n}\right|<\infty
$$

which yields the consequences that $\left\{p_{\infty}\right\}^{\alpha}=\left\{p_{c}\right\}^{\alpha}=\left\{p_{0}\right\}^{\alpha}=D$.

Theorem 3.5. Consider the sets $D_{1}, D_{2}$ and $D_{3}$ defined as follows:

$$
\begin{gathered}
D_{1}=\left\{a=\left(a_{k}\right) \in w: \sup _{n \in N} \sum_{k=0}^{n}\left|\sum_{i=k}^{n}(-1)^{i-k}\left(\begin{array}{c}
i \\
i-k
\end{array}\right) a_{i}\right|<\infty\right\}, \\
D_{2}=\left\{a=\left(a_{k}\right) \in w: \sum_{i=k}^{\infty}(-1)^{i-k}\left(\begin{array}{c}
i \\
i-k
\end{array}\right) a_{i} \text { exists for each } k \in \mathbb{N}\right\},
\end{gathered}
$$

and

$$
D_{3}=\left\{a=\left(a_{k}\right) \in w: \lim _{n \rightarrow \infty} \sum_{k=0}^{n} \sum_{i=k}^{n}(-1)^{i-k}\left(\begin{array}{c}
i \\
i-k
\end{array}\right) a_{i} \text { exists }\right\}
$$

Then $\left\{p_{0}\right\}^{\beta}=D_{1} \cap D_{2},\left\{p_{c}\right\}^{\beta}=D_{1} \cap D_{2} \cap D_{3}$ and $\left\{p_{\infty}\right\}^{\beta}=D_{2} \cap D_{3}$.

Proof. We give the proof only for the space $p_{0}$. Since the proof may be given by a similar way for the spaces $p_{c}$ and $p_{\infty}$, we omit it. Consider the equation

$$
\sum_{k=0}^{n} a_{k} x_{k}=\sum_{k=0}^{n}\left[\sum_{i=k}^{n}(-1)^{i-k}\left(\begin{array}{c}
i \\
i-k
\end{array}\right) y_{i}\right] a_{k}=\sum_{k=0}^{n}\left[\sum_{i=k}^{n}(-1)^{i-k}\left(\begin{array}{c}
i \\
i-k
\end{array}\right) a_{i}\right] y_{k}=(D y)_{n}
$$

where 


$$
D=\left(d_{n k}\right)=\left\{\begin{array}{c}
\sum_{i=k}^{n}(-1)^{i-k}\left(\begin{array}{c}
i \\
i-k
\end{array}\right) a_{i},(0 \leq k \leq n) \\
0,(k>n)
\end{array},(n, k \in \mathbb{N})\right.
$$

Thus, we deduce from Lemma 3.2 with (3.5) that $a x=\left(a_{k} x_{k}\right) \in c s$ whenever $x=\left(x_{k}\right) \in p_{0}$ if and only if $D y \in c$ whenever $y=\left(y_{k}\right) \in$ $c_{0}$. Therefore, using relations (3.2) and (3.3), we conclude that $\lim _{n \rightarrow \infty} d_{n k}$ exists fo each $k \in \mathbb{N}$ and

$$
\sup _{n \in \mathbb{N}} \sum_{k=0}^{n}\left|\sum_{i=k}^{n}(-1)^{i-k}\left(\begin{array}{c}
i \\
i-k
\end{array}\right) a_{i}\right|<\infty
$$

which shows that $\left\{p_{0}\right\}^{\beta}=D_{1} \cap D_{2}$.

Theorem 3.6. The $\gamma-$ dual of the sequence spaces $p_{\infty}, p_{c}$ and $p_{0}$ are $D_{1}$.

Proof. We give the proof only for the space $p_{0}$. Consider the equality

$$
\begin{aligned}
\left|\sum_{k=0}^{n} a_{k} x_{k}\right| & =\left|\sum_{k=0}^{n} a_{k}\left[\sum_{i=0}^{k}(-1)^{k-i}\left(\begin{array}{c}
k \\
k-i
\end{array}\right) y_{i}\right]\right| \\
& =\left|\sum_{k=0}^{n}\left[\sum_{i=k}^{n}(-1)^{i-k}\left(\begin{array}{c}
i \\
i-k
\end{array}\right) a_{i}\right] y_{k}\right| \\
& \leq \sum_{k=0}^{n}\left|\sum_{i=k}^{n}(-1)^{i-k}\left(\begin{array}{c}
i \\
i-k
\end{array}\right) a_{i}\right|\left|y_{k}\right| .
\end{aligned}
$$

Taking supremum over $n \in \mathbb{N}$, we get

$$
\begin{aligned}
\sup _{n \in N}\left|\sum_{k=0}^{n} a_{k} x_{k}\right| & \leq \sup _{n \in N}\left(\sum_{k=0}^{n}\left|\sum_{i=k}^{n}(-1)^{i-k}\left(\begin{array}{c}
i \\
i-k
\end{array}\right) a_{i}\right|\left|y_{k}\right|\right) \\
& \leq\|y\|_{c_{0}} \sup _{n}\left(\sum_{k=0}^{n}\left|\sum_{i=k}^{n}(-1)^{i-k}\left(\begin{array}{c}
i \\
i-k
\end{array}\right) a_{i}\right|\right) \leq \infty .
\end{aligned}
$$

This means that $a=\left(a_{k}\right) \in\left\{p_{0}\right\}^{\gamma}$. Hence,

$$
D_{1} \subset\left\{p_{0}\right\}^{\gamma}
$$

Conversely, let $a=\left(a_{k}\right) \in\left\{p_{0}\right\}^{\gamma}$ and $x \in p_{0}$. Then one can easily see that

$$
\left(\sum_{k=0}^{n}\left[\sum_{i=k}^{n}(-1)^{i-k}\left(\begin{array}{c}
i \\
i-k
\end{array}\right) a_{i}\right] y_{k}\right) \in l_{\infty}
$$

whenever $a x=\left(a_{k} x_{k}\right) \in b s$. This implies that the matrix $D$ given at the (3.6) is in the class $\left(c_{0}: l_{\infty}\right)$. Hence, the condition

$$
\sup _{n}\left(\sum_{k=0}^{n}\left|\sum_{i=k}^{n}(-1)^{i-k}\left(\begin{array}{c}
i \\
i-k
\end{array}\right) a_{i}\right|\right)<\infty
$$

is satisfied, which implies that $a=\left(a_{k}\right) \in D_{1}$. In other words,

$$
\left\{p_{0}\right\}^{\gamma} \subset D_{1}
$$

Therefore, by combining inclusions (3.7) and (3.8), we estahlish that the $\gamma$-dual of the sequence spaces $p_{0}$ is $D_{1}$, which completes the proof. 


\section{Some matrix mappings related to Pascal sequence spaces}

Lemma 4.1. [16, p. 57] The matrix mappings between BK-spaces are continuous.

Lemma 4.2. [16, $p .128] A \in\left(c: l_{p}\right)$ if and only if

$$
\sup _{K \in F} \sum_{n}\left|\sum_{k \in K} a_{n k}\right|^{p}<\infty, 1 \leq p<\infty .
$$

Theorem 4.3. $A \in\left(p_{c}: l_{p}\right)$ if and only if the following conditions are satisfied: For $1 \leq p<\infty$,

$$
\begin{gathered}
\sup _{K \in F} \sum_{k}\left|\sum_{k \in K} \sum_{i=k}^{n}(-1)^{i-k}\left(\begin{array}{c}
i \\
i-k
\end{array}\right) a_{n i}\right|^{p}<\infty, \\
\sum_{i=k}^{n}(-1)^{i-k}\left(\begin{array}{c}
i \\
i-k
\end{array}\right) a_{n i} \text { exists for all } k, n \in \mathbb{N}, \\
\sum_{k} \sum_{i=k}^{n}(-1)^{i-k}\left(\begin{array}{c}
i \\
i-k
\end{array}\right) a_{n i} \text { converges for all } n \in \mathbb{N}, \\
\sup _{m \in N} \sum_{k=0}^{m}\left|\sum_{i=k}^{m}(-1)^{i-k}\left(\begin{array}{c}
i \\
i-k
\end{array}\right) a_{n i}\right|<\infty, n \in \mathbb{N},
\end{gathered}
$$

and for $p=\infty$, conditions (4.3) and (4.5) are satisfied and

$$
\sup _{n \in \mathbb{N}} \sum_{k=0}^{n}\left|\sum_{i=k}^{n}(-1)^{i-k}\left(\begin{array}{c}
i \\
i-k
\end{array}\right) a_{n i}\right|<\infty .
$$

Proof. Let $1 \leq p<+\infty$. Assume that conditions (4.2) - (4.6) are satisfied and take any $x \in p_{c}$. Then $\left(a_{n k}\right) \in\left(p_{c}\right)^{\beta}$ for all $k, n \in \mathbb{N}$, which implies that $A x$ exists. We define the matrix $G=\left(g_{n k}\right)$ with

$$
g_{n k}=\sum_{i=k}^{n}(-1)^{i-k}\left(\begin{array}{c}
i \\
i-k
\end{array}\right) a_{n i}
$$

for all $k, n \in \mathbb{N}$. Then, since condition (4.1) is satisfied for the matrix $G$, we have $G \in\left(c: l_{p}\right)$. Now consider the following equality obtained from the s. th partial sum of the series $\sum_{k} a_{n k} x_{k}$ :

$$
\sum_{k=0}^{s} a_{n k} x_{k}=\sum_{k=0}^{s} \sum_{i=k}^{s}(-1)^{i-k}\left(\begin{array}{c}
i \\
i-k
\end{array}\right) a_{n i} y_{k}, m, n \in \mathbb{N} .
$$

Therefore, we derive from (4.7) as $s \rightarrow \infty$ that

$$
\sum_{k=0}^{\infty} a_{n k} x_{k}=\sum_{k=0}^{\infty} \sum_{i=k}^{n}(-1)^{i-k}\left(\begin{array}{c}
i \\
i-k
\end{array}\right) a_{n i} y_{k}, n \in \mathbb{N} .
$$

Whence taking $l_{p}$-norm we get

$$
\|A x\|_{l_{p}}=\|G y\|_{l_{p}}<\infty .
$$

This means that $A \in\left(p_{c}: l_{p}\right)$. Now let $p=\infty$. Assume that conditions (4.2) - (4.6) are satisfied and take any $x \in p_{c}$. Then $\left(a_{n k}\right) \in\left(p_{c}\right)^{\beta}$ for all $k, n \in \mathbb{N}$, which implies that $A x$ exists. Whence taking $l_{\infty}$-norm (4.8)

$$
\|A x\|_{l_{\infty}}=\sup _{n \in N}\left|\sum_{k} g_{n k}\right| \leq\|y\|_{l_{\infty}} \sup _{n \in N} \sum_{k}\left|g_{n k}\right|<\infty .
$$

Then, we have $A \in\left(p_{c}: l_{\infty}\right)$.

Conversely, assume that $A \in\left(p_{c}: l_{p}\right)$. Then, since $p_{c}$ and $l_{p}$ are $B K$-spaces, it follows from Lemma 4 that there exists a real constant $K>0$ such that

$$
\|A x\|_{l_{p}}=K\|x\|_{h_{c}}
$$

for all $x \in p_{c}$. Since inequality (4.10) also holds for the sequence

$$
x=\left(x_{k}\right)=\sum_{k \in F} b^{(k)} \in p_{c}
$$


where

$$
b^{(k)}=\left\{b_{n}^{(k)}\right\}=\left\{\begin{array}{c}
0,(0 \leq n<k) \\
(-1)^{n-k}\left(\begin{array}{c}
n \\
n-k
\end{array}\right),(n \geq k
\end{array}\right.
$$

for every fixed $k \in \mathbb{N}$. We have

$$
\|A x\|_{l_{p}}=\left[\sum_{n}\left|\sum_{k \in F} \sum_{i=k}^{n}(-1)^{i-k}\left(\begin{array}{c}
i \\
i-k
\end{array}\right) a_{n i}\right|^{p}\right]^{\frac{1}{p}} \leq K\|x\|_{p_{c}}=K,
$$

which shows the necessity of (4.2).

Theorem 4.4. $A \in\left(p_{c}: c\right)$ if and only if conditions (4.3), (4.5) and (4.6) are satisfied,

$$
\lim _{n \rightarrow \infty} \sum_{i=k}^{n}(-1)^{i-k}\left(\begin{array}{c}
i \\
i-k
\end{array}\right) a_{n i}=\alpha_{k} \text { for all } k \in \mathbb{N}
$$

and

$$
\lim _{n \rightarrow \infty} \sum_{k} \sum_{i=k}^{n}(-1)^{i-k}\left(\begin{array}{c}
i \\
i-k
\end{array}\right) a_{n i}=\alpha .
$$

Proof. Assume that $A$ satisfies conditions (4.3), (4.5), (4.6), (4.11) and (4.12). Let us take an arbitrary an $x=\left(x_{k}\right)$ in $p_{c}$ such that $x_{k} \rightarrow l$ as $k \rightarrow \infty$. Then $A x$ exists, and it is trivial that the sequence $y=\left(y_{k}\right)$ associated with the sequence $x=\left(x_{k}\right)$ by relation (2.3) belongs to $c$ and is such that $y_{k} \rightarrow l$ as $k \rightarrow \infty$. At this stage, it follows from (4.11) and (4.6) that

$$
\sum_{j=0}^{k}\left|\alpha_{j}\right| \leq \sup _{n \in N} \sum_{j}\left|\sum_{i=k}^{n}(-1)^{i-k}\left(\begin{array}{c}
i \\
i-k
\end{array}\right) a_{n i}\right|<\infty
$$

for every $n \in \mathbb{N}$. This yield $\alpha_{n} \in l_{1}$. Considering (4.8), we write

$$
\sum_{k} a_{n k} x_{k}=\sum_{k} \sum_{i=k}^{n}(-1)^{i-k}\left(\begin{array}{c}
i \\
i-k
\end{array}\right) a_{n i}\left(y_{k}-l\right)+l \sum_{k} \sum_{i=k}^{n}(-1)^{i-k}\left(\begin{array}{c}
i \\
i-k
\end{array}\right) a_{n i} y_{k} .
$$

In this situation, letting $n \rightarrow \infty$ in (4.13), we establish that the first term on the right-hand side tends to $\sum_{k} \alpha_{k}\left(y_{k}-l\right)$ by (4.6) and(4.11), and the second term tends to $l \alpha$ by (4.11). Taking these facts into account, we deduce from (4.13) as $n \rightarrow \infty$ that

$$
(A x)_{n} \rightarrow \sum_{k} \alpha_{k}\left(y_{k}-l\right)+l \alpha
$$

which shows that $A \in\left(p_{c}: c\right)$.

Conversely, assume that $A \in\left(p_{c}: c\right)$. Then, since the inclusion $c \subset l_{\infty}$ holds the necessity of (4.3), (4.5) and (4.6) is immediately obtained from

$$
\sup _{n} \sum_{k}\left|\sum_{i=k}^{n}(-1)^{i-k}\left(\begin{array}{c}
i \\
i-k
\end{array}\right) a_{n i}\right|<\infty .
$$

To prove the necessity of (4.11) consider the sequence $x=b^{(k)}=\left\{b_{n}^{(k)}\right\}_{n \in \mathbb{N}}$ in $p_{c}$. Where

$$
b^{(k)}=\left\{b_{n}^{(k)}\right\}=\left\{\begin{array}{c}
0,(0 \leq n<k) \\
(-1)^{n-k}\left(\begin{array}{c}
n \\
n-k
\end{array}\right),(n \geq k
\end{array}\right.
$$

for every fixed $k \in \mathbb{N}$. Since $A x$ exists and belongs to $c$ for every $x \in p_{c}$, one can easily see that

$$
A b^{(k)}=\left\{\sum_{i=k}^{n}(-1)^{i-k}\left(\begin{array}{c}
i \\
i-k
\end{array}\right) a_{n i}\right\}_{n \in \mathbb{N}}
$$

for each $k \in \mathbb{N}$, which yields the necessity of (4.11).

Similarly, by setting $x=e=(1,1, \ldots)$ in $(4.8)$, we obtain

$$
A x=\left\{\sum_{k} \sum_{i=k}^{n}(-1)^{i-k}\left(\begin{array}{c}
i \\
i-k
\end{array}\right) a_{n i}\right\}_{n \in \mathbb{N}},
$$

which belongs to the space $c$, and this shows the necessity of (4.12). This step conludes the proof. 


\section{References}

[1] B. Choudhary and S. Nanda, Functional Analysis with Applications, Wiley, New Delhi, 1989.

[2] M. Kirişçi, On the Taylor Sequence Spaces of Non-Absolute Type which Include The Spaces $c_{0}$ and c, Journal of Math. Analysis 6 (2015), $22-35$.

[3] B. Altay, F. Başar, Some Euler Sequence Spaces of Non-Absolute Type, Ukrainian Math. J. 57 (2005), 1-17. [4] M. Şengönül, F. Başar, Some New Cesaro Sequence Spaces of Non-Absolute Type which Include The Spaces $c_{0}$ and c, Soochow J. Math. 31 (2005),
107-119.

[5] B. Altay, F. Başar, M. Mursaleen, On the Euler sequence spaces which include in the spaces $l_{p}$ and $l_{\infty}$, Inform. Sci. 176 (2006), $1450-1462$.

[6] E. Malkowsky, Recent results in the theory of matrix transformations in sequences spaces, Mat. Vesnik 49 (1997), 187-196.

[7] P. N. Ng, P. Y. Lee, Cesaro sequences spaces of non-absolute type, Comment. Math. Prace Mat. 20 (1978), 429-433.

[8] C. S. Wang, On Nörlund seqence spaces, Tamkang J. Math. 9 (1978), 269-274.

[9] G. H. Lawden, Pascal matrices, Mathematical Gazette 56 (1972), 325-327.

[10] S. Dutta and P. Baliarsingh, On some Toeplitz matrices and their inversions, J. Egypt Math. Soc. 22 (2014), $420-423$.

[11] R. Brawer, Potenzen der Pascal matrix und eine Identitat der Kombinatorik, Elem. der Math. 45 (1990), 107-110.

[12] A. Edelman and G. Strang, Pascal Matrices, The Mathematical Association of America, Monthly 111 (2004), $189-197$.

[13] C. Lay David, Linear Algebra and Its Applications, 4th Ed. Boston, Pearson, Addison-Wesley, 2012.

[14] I. J. Maddox, Elements of Functional Analysis, Cambridge University Press, Cambridge, 1981.

[15] D.J.H. Garling, The $\alpha-, \beta-$ and $\gamma-$ Duality of Sequence Spaces, Proc. Comb. Phil. Soc. 63 (1967), 963-981.

[16] M. Stieglitz, H. Tietz, Matrixtransformationen von Folgenraumen Eine Ergebnisübersict, Math. Z. 154 (1977), 1-16. 